\title{
Mercury Exposure and Heart Rate Variability: a Systematic Review
}

\author{
Matthew O. Gribble ${ }^{1}$ Alan Cheng ${ }^{2} \cdot$ Ronald D. Berger ${ }^{2} \cdot$ Lori Rosman $^{3}$. \\ Eliseo Guallar ${ }^{4,5}$
}

Published online: 26 June 2015

(C) Springer International Publishing AG 2015

\begin{abstract}
Background Mercury affects the nervous system and has been implicated in altering heart rhythm and function. We sought to better define its role in modulating heart rate variability, a well-known marker of cardiac autonomic function.

Design This is a systematic review study.

Methods We searched PubMed, Embase, TOXLINE, and DART databases without language restriction. We report findings as a qualitative systematic review because heterogeneity in study design and assessment of exposures and outcomes across studies, as well as other methodological limitations of the literature, precluded a quantitative meta-analysis.

Results We identified 12 studies of mercury exposure and heart rate variability in human populations (ten studies involving primarily environmental methylmercury exposure and two
\end{abstract}

This article is part of the Topical Collection on Metals and Health

Electronic supplementary material The online version of this article (doi:10.1007/s40572-015-0053-0) contains supplementary material, which is available to authorized users.

Eliseo Guallar

eguallar@jhu.edu

1 Department of Preventive Medicine, University of Southern California, Los Angeles, CA, USA

2 Division of Cardiology, Johns Hopkins School of Medicine, Baltimore, MD, USA

3 Welch Medical Library, Johns Hopkins School of Medicine, Baltimore, MD, USA

4 Department of Epidemiology, Johns Hopkins Bloomberg School of Public Health, Baltimore, MD, USA

5 Welch Center for Prevention, Epidemiology and Clinical Research, Johns Hopkins Medical Institutions, 2024 E. Monument St., Room 2-645, Baltimore, MD 21205, USA studies involving occupational exposure to inorganic mercury) conducted in Japan, the Faroe Islands, Canada, Korea, French Polynesia, Finland, and Egypt. The association of prenatal mercury exposure with lower high-frequency band scores (thought to reflect parasympathetic activity) in several studies, in particular the inverse association of cord blood mercury levels with the coefficient of variation of the R-R intervals and with lowfrequency and high-frequency bands at 14 years of age in the Faroe Islands birth cohort study, suggests that early mercury exposure could have a long-lasting effect on cardiac parasympathetic activity. Studies with later environmental exposures to mercury in children or in adults were heterogeneous and did not show consistent associations.

Conclusions The evidence was too limited to draw firm causal inferences. Additional research is needed to elucidate the effects of mercury on cardiac autonomic function, particularly as early-life exposures might have lasting impacts on cardiac parasympathetic function.

Keywords Mercury $\cdot$ Heart rate variability $\cdot$ Cardiac autonomic function $\cdot$ Neurotoxicity $\cdot$ Pediatric neurotoxicity

\section{Introduction}

Mercury is a well-established neurotoxicant $[1,2]$, with particularly harmful effects during neurodevelopment in utero and early childhood [3, 4]. Questions remain regarding other possible biological effects of mercury, including possible associations with diseases of the cardiovascular system including hypertension, coronary heart disease, and myocardial infarction $[4,5 \cdot \bullet]$. As heart rhythm and function are under autonomic nervous system control, it has been hypothesized that the neurotoxic effects of mercury might also impact cardiac autonomic function [6]. 
Heart rate variability is a widely used measure of cardiac autonomic function. Most heart rate variability parameters fall into two common categories: time domain metrics, which describe beat-to-beat variation, and frequency domain measures, which examine the heartbeat pattern via spectral analysis [7]. The most common frequency domain measures are highfrequency band (HF), often interpreted as a measure of parasympathetic activity, low-frequency band (LF), often interpreted as a mixture of parasympathetic and sympathetic activity, and LF/HF ratio, often interpreted as a measure of sympathetic activity unless HF is reduced [7]. The specific biological interpretation of the heart rate variability parameters is open to some debate [8], but heart rate variability measures are generally regarded as reflecting cardiac autonomic nervous system functioning. Heart rate variability is a strong predictor of mortality after acute myocardial infarction [9-13] and may predict mortality after stroke [14] and ventricular tachyarrythmias [9].

The objective of this systematic review was to consolidate available evidence of the association between mercury exposure and heart rate variability measures in human populations. We separated our review into general population studies and occupational studies given differences in the type of mercury encountered by the general population (methylmercury from fish $\left[15,16^{\bullet}\right]$ ) and certain occupational groups (inorganic mercury [17]).

\section{Methods}

We focused our search on the association between mercury exposures and cardiovascular disease outcomes in human population studies. We used the bibliography from a US Environmental Protection Agency panel evaluating the cardiovascular effects of methylmercury exposure [5••] to develop a set of keywords and controlled vocabulary terms for querying PubMed, Embase, TOXLINE, and DART databases (see Appendix 1). We followed best practices for our search strategy [18], including database-tailored search terms, appropriate search headings, and consideration of alternate spellings of key terms. No language or date restrictions were applied. The search strategy resulted in 8514 unique citations as of June 2, 2014.

We reviewed the title and available abstracts of all references to identify studies of mercury and cardiovascular disease, followed by full-text review of potentially relevant or unclear papers (Fig. 1). Foreign-language articles were translated if necessary. We excluded papers lacking measures of mercury exposure (biomarkers, environmental measures, or indirect measures such as Minamata disease diagnosis); papers lacking measures of heart rate variability; autopsy studies; case reports, case series, editorials, letters, and review papers; and studies not on humans.



Fig. 1 Study selection. Potentially relevant citations were identified in four databases (PubMed, Embase, TOXLINE, and DART) and filtered to a set of 12 papers on the epidemiology of mercury exposure and heart rate variability outcomes

Although we had originally planned to combine the results of the studies in a quantitative meta-analysis, it was deemed inappropriate due to methodological limitations of the literature and heterogeneity in study design, exposure assessment and outcome assessment across studies. We therefore summarized results narratively within groups of similar populations.

\section{Results}

We identified 12 studies of mercury exposure and heart rate variability in human populations [19-30]. The characteristics of these studies are summarized in Table 1. Ten studies involved primarily environmental methylmercury exposure while two studies involved occupational exposure to inorganic mercury. Eleven studies were observational, while one study was a nonrandomized trial of fish intake. The studies were conducted in Japan, the Faroe Islands, Canada (Inuit and Cree communities), Korea, French Polynesia, Finland, and Egypt. Three studies included only children or adolescents; seven studies included only adults; and two studies included both age groups. Only one study explicitly excluded participants with heart disease or medication use that may affect heart rate variability.

Mercury exposure was assessed in blood, urine, toenails, hair, umbilical cord blood, or as fetal Minamata disease status (Table 1). Four studies had biomarkers of fish oil levels, and two other studies had dietary information on fish intake. There was also substantial heterogeneity in the heart rate variability outcomes reported (Table 2). The most common time domain metrics reported were the standard deviation of the R-R intervals (SDNN) and the coefficient of variation of the R-R intervals (CVRR), while the most common frequency domain 





metrics reported were LF, HF, and the LF/HF ratio. Studies were also heterogeneous in the measurement interval. Six studies used 5-min ECG recordings or $300 \mathrm{R}-\mathrm{R}$ intervals; four studies used 2-h ECG recordings; and two studies used a 1min recording or $60 \mathrm{R}-\mathrm{R}$ intervals. Only two studies reported the time of day during heart rate variability sampling.

\section{Studies of Environmental Mercury Exposure}

\section{Studies in Children or Adolescents}

The 14-year follow-up visit of the Faroe Islands birth cohort $(N=857)$ measured mercury in cord blood and in hair at 7 and 14 years of age and measured heart rate variability at ages 7 and 14. Cord blood mercury was inversely associated with CVRR at age 14; on the other hand, no association between hair mercury and CVRR at ages 7 or 14 was observed (Table 3). In this study, the association of mercury biomarkers with LF and HF domains were all inverse but were only statistically significant for cord blood mercury and LF at seven and 14 years of age, for hair mercury and LF at 7 years of age, and for cord blood and HF at 14 years of age (Table 4). The associations between mercury biomarkers and LF/HF ratio did not reach statistical significance.

Murata et al. studied 136 Japanese children and found significant positive associations of cord tissue and mercury hair with LF, nonsignificant positive associations with LF/HF (Table 4), and a significant inverse association of cord tissue mercury with log-CCV-LF. Valera et al. conducted two crosssectional studies of 78 adolescents in French Polynesia and of 226 Inuit children 11 years of age in Northern Quebec, Canada. The study in French Polynesia showed no consistent trends between blood mercury and time or frequency domain measures. Among Inuit children, the associations between mercury measured in cord blood, blood, or hair and time or frequency domain measures were virtually all inverse, and were statistically significant for blood mercury and SDNN, SDANN, CVRR, and LF (Tables 3 and 4). Finally, the cohort study of children and adults by Lim et al. found a decrease in $\mathrm{HF}$ in the first generation of life in the Banwol region of Korea and the second generation of life in the Swiha region (Table 4).

\section{Studies in Adults}

Oka et al. compared nine adults with fetal Minimata disease to 13 age-matched controls and found lower SDNN, CVRR, LF, and HF in cases compared to controls, although only the difference for HF was statistically significant. The Korean cohort study by Lim et al. also presented results combining patients across all ages (largely adult participants) and found negative point estimate associations with $\log (\mathrm{SDNN}), \mathrm{LF}$ and HF, although only HF was significant (Tables 3 and 4). Valera et al. conducted cross-sectional studies in adults including 205 Inuit and 663 Cree adults in Northern Quebec, and 146 adults in French Polynesia. Among Inuits, blood mercury was inversely related to all time domain measures, although the associations were statistically significant only for SDANN. The associations with frequency domains were virtually null. Among Cree adults, blood or hair mercury showed no statistically significant associations with time domain measures but showed significant positive associations with LF, HF, and LF/ HF. The study in French Polynesian adults showed no consistent trends between blood mercury and time or frequency domain measures.

A small cross-sectional study of Faroese whalers $(N=42)$ found positive associations between several biomarkers of mercury exposure and CVRR, C-CVLF, and C-CVHF, with significant associations for blood mercury and CVRR, CCVLF, and C-CVHF, and for nail and hair mercury with CCVLF. In a cross-sectional study of 1589 men and women living near an industrial complex in South Korea, Lim et al. found inverse associations between hair mercury and SDNN, LF, and HF, but only the association with HF was statistically significant.

Yaginuma-Suraki et al. conducted the only clinical trial identified in our search. They studied 27 participants who consumed methylmercury-containing bigeye tuna or swordfish once a week for 14 weeks and compared them to 27 other subjects who were instructed to continue their usual diets. At the end of the intervention period, participants in the intervention group had higher levels of CVRR, LF, and LF/HF compared with controls. The difference for LF/HF was statistically significant.

\section{Studies of Occupational Mercury Exposure}

Two studies evaluated heart rate variability in mercuryexposed occupational populations. Piikivi studied 41 chloralkali plant workers and 41 unexposed controls using 60 consecutive R-R. Abdel-Hamid et al. studied 30 lamp factory workers and 20 unexposed workers from a food industrial plant using a 1-min ECG recording. These studies did not report standard measures of heart rate variability (Table 2).

\section{Discussion and Conclusion}

We identified 12 population studies of mercury exposures and heart rate variability outcomes, including environmental studies of children/adolescents and adults, and occupational studies. There was marked heterogeneity across the studies in study design, study population, assessment of mercury exposure, ECG recording methodology, and heart rate variability metrics reported. The inverse association of cord blood mercury levels with CVRR, and with LF and HF bands at 7 and 14 years of age in the cohort study of Faroe Islands children by 
Table 2 Heart rate variability ascertainment methods and reporting

\begin{tabular}{|c|c|c|c|c|c|c|c|c|c|c|}
\hline \multirow[t]{2}{*}{ First author, year } & \multirow[t]{2}{*}{ ECG duration } & \multicolumn{5}{|c|}{ Time domains } & \multicolumn{4}{|c|}{ Frequency domains } \\
\hline & & SDNN & SDANN & rMSSD & CVRR & Other & $\mathrm{LF}$ & $\mathrm{HF}$ & $\begin{array}{l}\mathrm{LF} / \\
\mathrm{HF}\end{array}$ & Other \\
\hline \multicolumn{11}{|l|}{ Environmental exposure } \\
\hline Oka, 2003 & $5 \mathrm{~min}$ & $\mathrm{X}$ & & & $\mathrm{X}$ & & $\mathrm{X}$ & $\mathrm{X}$ & & \\
\hline Grandjean, 2004 & 300 R-R intervals & & & & $\mathrm{X}$ & & $\mathrm{X}$ & $\mathrm{X}$ & $\mathrm{X}$ & CCV-LF, CCV-HF \\
\hline Murata, 2006 & 300 R-R intervals & & & & & & $\mathrm{X}$ & & $\mathrm{X}$ & $\begin{array}{l}\text { CCV-LF, CCV-HF, } \\
\text { PSD-LF, PSD-HF }\end{array}$ \\
\hline Valera, 2008 & $2 \mathrm{~h}$ & $\mathrm{X}$ & $\mathrm{X}$ & $\mathrm{X}$ & $\mathrm{X}$ & pNN50 & $\mathrm{X}$ & $\mathrm{X}$ & $\mathrm{X}$ & VLF \\
\hline Choi, 2009 & $5 \mathrm{~min}$ & & & & $\mathrm{X}$ & & & & & CCV-LF, CCV-HF \\
\hline Lim, 2010 & $5 \mathrm{~min}$ & $\mathrm{X}$ & & & & & $\mathrm{X}$ & $\mathrm{X}$ & & $\mathrm{TP}$ \\
\hline $\begin{array}{l}\text { Yaginuma-Sakurai, } \\
2010\end{array}$ & $300 \mathrm{R}-\mathrm{R}$ intervals ${ }^{\mathrm{a}}$ & & & & $\mathrm{X}$ & & $\mathrm{X}$ & & $\mathrm{X}$ & $\begin{array}{l}\text { CCV-LF, CCV-HF, } \\
\text { PSD-LF, PSD-HF }\end{array}$ \\
\hline Valera, 2011a & $2 \mathrm{~h}$ & $\mathrm{X}$ & $\mathrm{X}$ & $\mathrm{X}$ & & & $\mathrm{X}$ & $\mathrm{X}$ & $\mathrm{X}$ & \\
\hline Valera, 2011b & $2 \mathrm{~h}$ & $\mathrm{X}$ & $\mathrm{X}$ & $\mathrm{X}$ & & & $\mathrm{X}$ & $\mathrm{X}$ & $\mathrm{X}$ & \\
\hline Valera, 2012 & $2 \mathrm{~h}$ & $\mathrm{X}$ & $\mathrm{X}$ & $\mathrm{X}$ & $\mathrm{X}$ & & $\mathrm{X}$ & $\mathrm{X}$ & $\mathrm{X}$ & \\
\hline \multicolumn{11}{|l|}{ Occupational exposure } \\
\hline Piikivi, 1989 & $60 \mathrm{R}-\mathrm{R}$ intervals & & & & & $\begin{array}{l}\text { MSDD during normal } \\
\text { breathing, and maximal/ } \\
\text { minimal R-R during } \\
\text { deep breathing, during } \\
\text { Valsalva maneuver } \\
\text { and during fast tilting }\end{array}$ & & & & \\
\hline Abdel-Hamid, 2001 & $1 \mathrm{~min}$ & & & & & $\begin{array}{l}\text { Maximal R-R, minimal } \\
\text { R-R, maximal/minimal } \\
\text { R-R, (maximal-minimal) } \\
\text { /resting R-R }\end{array}$ & & & & \\
\hline
\end{tabular}

${ }^{a}$ Heart rate variability analyses based on 100 consecutive R-R intervals with the minimal standard deviation

$C C V$ component coefficient of variation, $C V R R$ coefficient of variation of R-R intervals, $p N N 50$ proportion of interval differences of successive R-R intervals $>50 \mathrm{~ms}, P S D$ power spectral density, $r M S S D$ square root of the mean square differences of successive R-R intervals, $S D A N N$ standard deviation of the average RR-intervals calculated over 5-min periods, $S D N N$ standard deviation of R-R intervals, $V L F$ very low frequency, $T P$ total power

Grandjean et al. suggest that early mercury exposure may have a long-lasting effect on autonomic heart activity. Studies with later environmental exposures to mercury in children or in adults were heterogeneous and did not show consistent associations between mercury exposure and heart rate variability parameters. Data on the association of occupational mercury exposure and heart rate variability were very limited and could not be used to draw epidemiological inferences. Overall, the evidence for a causal effect of mercury exposure on heart rate variability was limited and large high-quality and adequately standardized studies in populations with a wide range of mercury exposures are needed.

Perhaps the main limitation of the studies of mercury and heart rate variability was the lack of standardized methods for performing and reporting heart rate variability measurements. Heart rate variability measurements depend on duration of assessment and time of day. The standards developed by the Task Force of the European Society of Cardiology and the North American Society of Pacing and Electrophysiology recommend either a 5-min recording for frequency domain metrics or a 24-h recording for time domain metrics [31]. None of the studies in this review used 24-h ECG monitoring. The differences in recording intervals and times of the day at measurement across studies limited the comparability of the findings [31]. Furthermore, there were numerous heart rate variability metrics reported, opening the possibility of multiple comparison and selective reporting biases. Indeed, we found substantial heterogeneity in the heart variability metrics reported across studies in our review. Under these circumstances, clearly defined analytical protocols and replication of study findings are essential to establish associations between mercury exposures and cardiac autonomic function.

In addition, a major limitation of many studies was the lack of data on fish intake and biomarkers of fish oils. Fish oils may have important cardiovascular benefits [32-34], including impacts on heart rate variability [35-37]. Fish intake is the common source of both fish oils and methylmercury, so it is likely that fish oils may confound the association between methylmercury and heart rate variability parameters. This is 


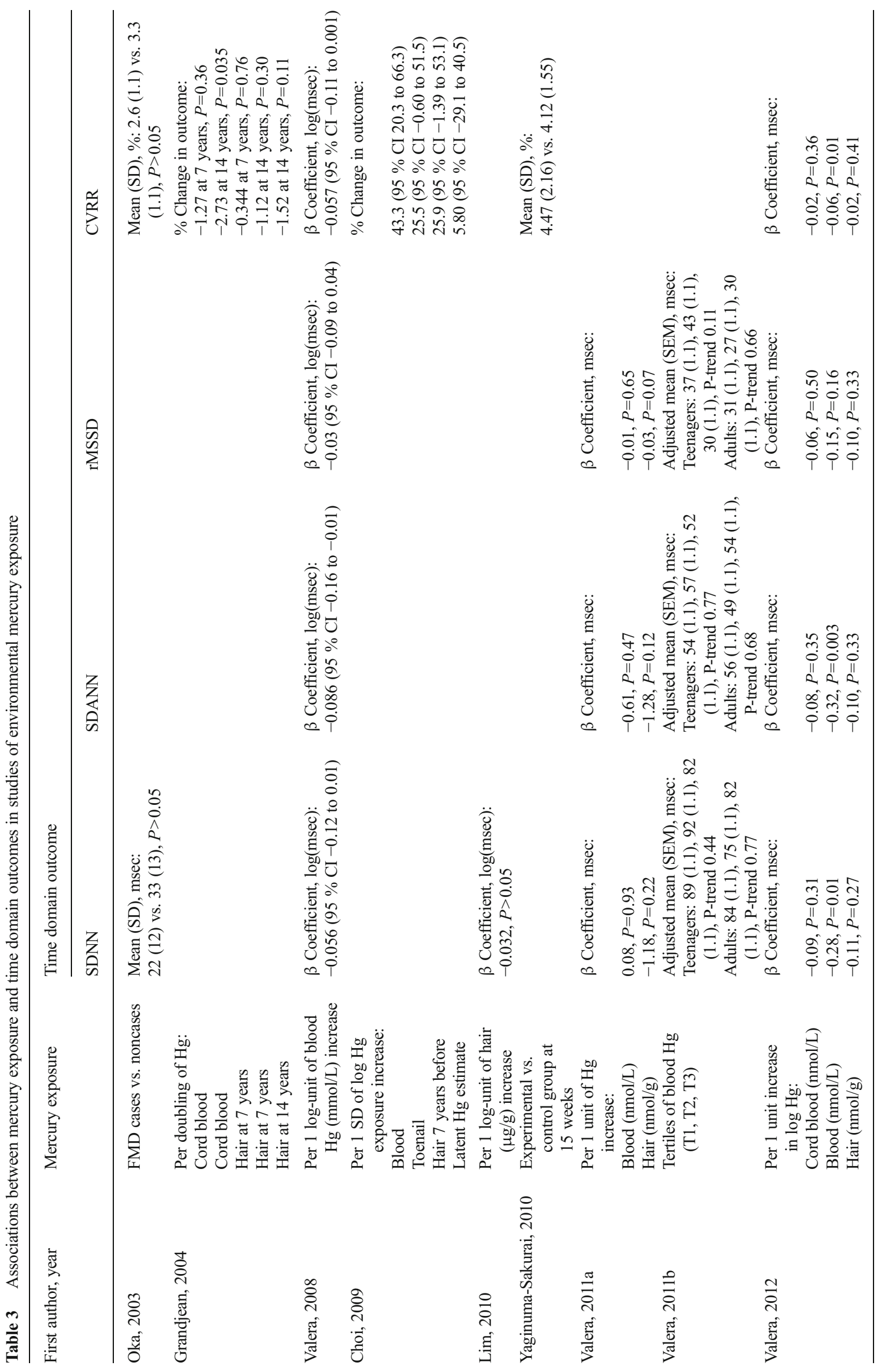




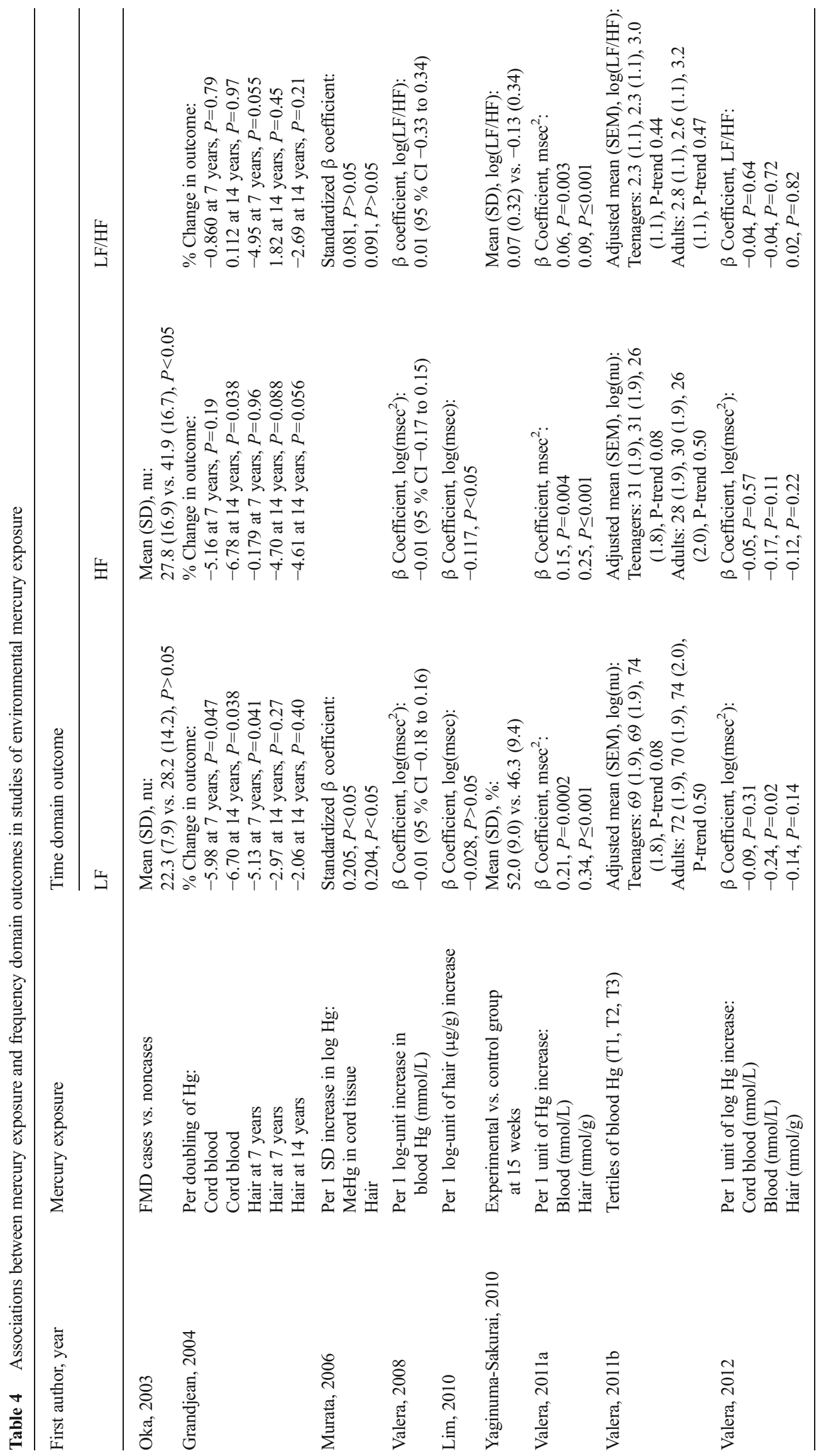


consistent with the confounding role of fish oils in other cardiovascular and neurological outcomes [38].

Two strategies to reduce confounding by fish oils are the collection of fish intake via dietary questionnaires and the measurement of biomarkers of fish oils. The accuracy of food frequency questionnaires in summarizing fish oil intake is somewhat limited [39-42], but studies with mercury and fish oil biomarkers and heart rate variability outcomes are rare. Indeed, the studies conducted by Valera et al. $(2008,2011 \mathrm{a}$, $2011 \mathrm{~b}$, and 2012) were the only observational studies in the review that measured and adjusted for biomarkers of fish oil intake.

Other methodological limitations common to many studies also deserve comment. Many studies were relatively small, and often several heart rate variability parameters showed changes consistent with mercury effects, but the associations were not statistically significant; thus, the findings were in many cases inconclusive. Heart rate variability is under complex physiological regulation and subject to substantial within person variability and to the influence of many external exposures. Epidemiological studies of the association of mercury with heart rate variability thus need adequate sample sizes to reliably identify potential associations. In addition, most studies were cross-sectional and did not allow for an evaluation of changes in heart rate variability parameters with changes in mercury exposure. Finally, many studies did not consider the presence of cardiovascular disease or the use of medications that may affect heart rate variability in study participants. Future studies should overcome these methodological limitations in order to obtain more precise and consistent estimates of the association of mercury exposure with heart rate variability.

Clinical trials of fish intake may address confounding and selection bias related to methylmercury intake. This was the approach taken by Yaginuma-Sakurai et al., although the trial was not randomized. In this trial, the investigators used muscle portions (not including fatty portions) of bigeye tuna and swordfish to provide mercury exposure with only limited amounts of fish oils to the intervention group. In addition to ethical issues in conducting a dietary intervention study, the selection of an adequate control group is challenging as fish oil intake may still be different between the intervention and control groups. In this study, participants in the intervention group were instructed restrict their consumption of contaminated fish (other than the fish provided by the study), while participants in the control group were instructed to continue their usual diets. As a consequence, fish oil intake decreased in the intervention group but stayed constant in the control group, complicating the interpretation of the findings. The main finding of the trial was a higher LF band and LF/HF ratio among participants in the intervention group that Yaginuma-Sakurai et al. attributed to a sympathodominant state induced by methylmercury exposure. Replication of these findings in a larger sample size with appropriate randomization and control intervention would be important to establish the short-term effects of methylmercury on heart rate variability.

In the Faroe Islands' cohort, cord blood mercury levels were associated with decreased CVRR, LF and HF bands, although the associations were stronger at 14 years than at 7 years of follow-up. The inverse associations with LF and HF bands were interpreted as mercury-mediated decreases in sympathetic and parasympathetic modulation of heart rate variability. In this cohort, mercury exposure was also associated with brainstem auditory evoked potentials, and the authors speculated that the effects of mercury on heart rate variability could reflect brainstem neurotoxicity of mercury.

The biological mechanisms of mercury neurotoxicity are challenging to disentangle and are still under investigation, but may include oxidative damage, reversible thiol group binding, and interference with zinc-based metalloproteases required for neuronal development [43-45]. There is a growing literature on the immunological toxicity of mercury species [46-53], including recent evidence that toll-like receptor 4 might be affected by inorganic mercury in a vole animal model [54], and toll-like receptors 2 and 4 may influence heart rate variability in mice [55].

Mercury neurotoxicity may have several important modifiers in human populations including age, sex, and life stage of exposure [4]. Recently, epidemiological studies have explored gene-mercury interactions for human mercury neurotoxicity and identified possible genetic risk modifiers for mercury exposure [56-59]. Possible gene-environment interactions with mercury for heart rate variability outcomes might be explored in future research.

Both methylmercury and inorganic mercury are neurotoxic. The primary exposure to inorganic mercury in the general population is via mercury-containing amalgam in dental restorations. Workers, however, can be exposed to high levels of inorganic as mercury is used in numerous products and industrial processes, including the manufacture of industrial chemicals, in electrical and electronic applications, in fluorescent lamps, and in some cosmetics. Evaluating the cardiac autonomic effects of inorganic mercury exposure in workers is thus very important. However, we could only identify two studies of heart rate variability in mercury-exposed workers, but these studies used very short ECG recordings and unconventional heart rate variability metrics. Data on occupational mercury exposures and autonomic nervous function are lacking.

Heart rate variability is a noninvasive measure useful for summarizing cardiac autonomic pathology, in particular in community settings where more invasive procedures are infeasible [60]. It is easiest to interpret heart rate variability parameters when ascertained under standardized conditions [31]. Although measurable in community settings, it is not a 
perfect indication of cardiac autonomic function. For example, heart rate variability may have lower sensitivity than more invasive measures such as I-123 metaiodobenzylguanidine myocardial scintigraphy for detecting cardiac autonomic neuropathy among persons with diabetes [61]. It nevertheless has prognostic value for risk stratification in cardiovascular disease and related conditions like stroke, diabetes, and neurological disorders [8, 62]. However, it remains unclear whether diminution in heart rate variability via a direct neurotoxic effect carries the same prognostic implications as that seen when heart rate variability is diminished in the setting of myocardial disease.

In summary, in this systematic review, we found some evidence that mercury exposure might affect heart rate variability, particularly early exposures in children. This is consistent with other findings of early-life mercury neurological susceptibility [4]. If moderate-dose early-life mercury exposure has a cardiovascular autonomic neurotoxicity, this may have important public health implications for seafood-eating communities around the world [63•]. Overall, however, the evidence we identified is too limited and heterogeneous to draw causal inferences concerning the effect of mercury exposure on autonomic nervous function. Future studies should evaluate the effects of mercury on cardiac autonomic function, particularly in early exposures in pediatric populations. These studies should include adequate sample sizes, perform and report standardized heart rate variability measurements, use methods that appropriate adjust for potential confounding by fish oil intake in environmental mercury exposure, and evaluate the long term implications of mercury-affected heart rate variability on cardiovascular health.

Acknowledgments The authors are grateful to Andrew Menke for his assistance in the article review and abstraction process. The authors are also grateful to colleagues who assisted with foreign language manuscripts: Wilson Velasco (Russian, Japanese), Yiyi Zhang (Chinese), Marcia Varella (Portuguese), Kunihiro Matsushita (Japanese), Dimitrios Panagopoulos (Swedish), Sophia Jansson (Swedish), Ruth Wimsatt (Norweigan), Elizabeth Denning (German); and Johns Hopkins Medicine International Language Access Services (Korean, Italian, Croatian, Polish). Translations were paid for by institutional funds to Dr. Guallar. M. Gribble was supported during this project by T32 training grant support from the National Heart, Lung, and Blood Institute (5T32HL007024), the National Institute of Diabetes and Digestive and Kidney Diseases (5T32DK062707-10), and the National Institute for Environmental Health Sciences (T32ES013678-07).

\section{Compliance with Ethics Guidelines}

Conflict of Interest Matthew O. Gribble, Alan Cheng, Ronald D. Berger, Lori Rosman, and Eliseo Guallar declare that they have no conflict of interest.

Human and Animal Rights and Informed Consent This article does not contain any studies with human or animal subjects performed by any of the authors.

\section{References}

Papers of particular interest, published recently, have been highlighted as:

- Of importance

•- Of major importance

1. Poulin J, H. Gibb. Mercury: Assessing the environmental burden of disease at national and local levels. WHO Environmental Burden of Disease Series No. 16, A. Prüss-Üstün, Editor 2008, World Health Organization: Geneva.

2. Tchounwou PB et al. Environmental exposure to mercury and its toxicopathologic implications for public health. Environ Toxicol. 2003;18(3):149-75.

3. Counter SA, Buchanan LH. Mercury exposure in children: a review. Toxicol Appl Pharmacol. 2004;198(2):209-30.

4. Karagas MR et al. Evidence on the human health effects of lowlevel methylmercury exposure. Environ Health Perspect. 2012;120(6):799-806.

$5 . \bullet$ Roman HA et al. Evaluation of the cardiovascular effects of methylmercury exposures: current evidence supports development of a dose-response function for regulatory benefits analysis. Environ Health Perspect. 2011;119(5):607-14. This United States Environmental Protection Agency workshop review discusses much of the evidence of possible effects of mercury on the cardiovascular system.

6. Zhao D et al. A longitudinal study of age-related changes in intraocular pressure: the Kangbuk Samsung Health Study. Invest Ophthalmol Vis Sci. 2014;55(10):6244-50.

7. Kleiger RE, Stein PK, Bigger Jr JT. Heart rate variability: measurement and clinical utility. Ann Noninvasive Electrocardiol. 2005;10(1):88-101.

8. Nicolini $\mathrm{P}$ et al. The prognostic value of heart rate variability in the elderly, changing the perspective: from sympathovagal balance to chaos theory. Pacing Clin Electrophysiol. 2012;35(5):622-38.

9. Reed MJ, CE Robertson, PS Addison. Heart rate variability measurements and the prediction of ventricular arrhythmias. 2005; 98 : 87-95.

10. Kleiger RE et al. Decreased heart rate variability and its association with increased mortality after acute myocardial infarction. Am J Cardiol. 1987;59(4):256-62.

11. Malik M, et al. Heart rate variability in relation to prognosis after myocardial infarction: Selection of optimal processing techniques. 1989; 10: 1060-1074.

12. Wolf MM et al. Sinus arrhythmia in acute myocardial infarction. Med J Aust. 1978;2(2):52-3.

13. Bigger Jr JT et al. Frequency domain measures of heart period variability and mortality after myocardial infarction. Circulation. 1992;85(1):164-71.

14. Gujjar AR et al. Heart rate variability and outcome in acute severe stroke: role of power spectral analysis. Neurocrit Care. 2004;1(3): 347-53.

15. Selin NE et al. Sources of mercury exposure for U.S. seafood consumers: implications for policy. Environ Health Perspect. 2010;118(1):137-43.

16. Karimi R, Fitzgerald TP, Fisher NS. A quantitative synthesis of mercury in commercial seafood and implications for exposure in the United States. Environ Health Perspect. 2012;120(11):1512-9. This synthesizes data on exposure to mercury in seafood and is highly relevant for mercury exposure assessment.

17. Meyer-Baron M, Schaeper M, Seeber A. A meta-analysis for neurobehavioural results due to occupational mercury exposure. Arch Toxicol. 2002;76(3):127-36. 
18. Sampson $\mathrm{M}$ et al. An evidence-based practice guideline for the peer review of electronic search strategies. J Clin Epidemiol. 2009;62(9): 944-52.

19. Oka T et al. Autonomic nervous functions in fetal type Minamata disease patients: assessment of heart rate variability. Tohoku J Exp Med. 2002;198(4):215-21.

20. Grandjean P et al. Cardiac autonomic activity in methylmercury neurotoxicity: 14-year follow-up of a Faroese birth cohort. J Pediatr. 2004;144(2):169-76.

21. Murata $\mathrm{K}$ et al. Subclinical effects of prenatal methylmercury exposure on cardiac autonomic function in Japanese children. Int Arch Occup Environ Health. 2006;79(5):379-86.

22. Valera B, Dewailly E, Poirier P. Cardiac autonomic activity and blood pressure among Nunavik Inuit adults exposed to environmental mercury: a cross-sectional study. Environ Health. 2008;7: 29.

23. Choi AL et al. Methylmercury exposure and adverse cardiovascular effects in Faroese whaling men. Environ Health Perspect. 2009;117(3):367-72.

24. Lim S, Chung HU, Paek D. Low dose mercury and heart rate variability among community residents nearby to an industrial complex in Korea. Neurotoxicology. 2010;31(1):10-6.

25. Yaginuma-Sakurai $\mathrm{K}$ et al. Intervention study on cardiac autonomic nervous effects of methylmercury from seafood. Neurotoxicol Teratol. 2010;32(2):240-5.

26. Valera B, Dewailly E, Poirier P. Impact of mercury exposure on blood pressure and cardiac autonomic activity among Cree adults (James Bay, Quebec, Canada). Environ Res. 2011;111(8):1265-70.

27. Valera B et al. Influence of mercury exposure on blood pressure, resting heart rate and heart rate variability in French Polynesians: a cross-sectional study. Environ Health. 2011;10:99.

28. Valera B et al. Cardiac autonomic activity and blood pressure among Inuit children exposed to mercury. Neurotoxicology. 2012;33(5):1067-74.

29. Abdel-Hamid HA, Fahmy FC, Sharaf IA. Influence of free radicals on cardiovascular risk due to occupational exposure to mercury. J Egypt Public Health Assoc. 2001;76(1-2):53-69.

30. Piikivi L. Cardiovascular reflexes and low long-term exposure to mercury vapour. Int Arch Occup Environ Health. 1989;61(6):3915 .

31. Electrophysiology, T.F.o.t.E.S.o.C.t.N.A.S.o.P., Heart Rate Variability: Standards of Measurement, Physiological Interpretation, and Clinical Use. Circulation. 1996; 93(5): 10431065.

32. Xin W, Wei W, Li X. Effect of fish oil supplementation on fasting vascular endothelial function in humans: a meta-analysis of randomized controlled trials. PLoS ONE. 2012;7(9):e46028.

33. Xin W, Wei W, Li X. Effects of fish oil supplementation on cardiac function in chronic heart failure: a meta-analysis of randomised controlled trials. Heart. 2012;98(22):1620-5.

34. Wang Q et al. Effect of omega-3 fatty acids supplementation on endothelial function: a meta-analysis of randomized controlled trials. Atherosclerosis. 2012;221(2):536-43.

35. Xin W, Wei W, Li XY. Short-term effects of fish-oil supplementation on heart rate variability in humans: a meta-analysis of randomized controlled trials. Am J Clin Nutr. 2013;97(5):926-35.

36. Valera $\mathrm{B}$, et al. Influence of n-3 fatty acids on cardiac autonomic activity among Nunavik Inuit adults. Int J Circumpolar Health. 2012; 70(1)

37. Valera B. et al. Influence of Polyunsaturated Fatty Acids on Blood Pressure, Resting Heart Rate and Heart Rate Variability Among French Polynesians. J Am Coll Nutr. 2014: 1-9.

38. Choi AL et al. Negative confounding in the evaluation of toxicity: the case of methylmercury in fish and seafood. Crit Rev Toxicol. 2008;38(10):877-93.
39. Garneau V et al. Omega-3 fatty acids status in human subjects estimated using a food frequency questionnaire and plasma phospholipids levels. Nutr J. 2012;11:46.

40. Lucas $\mathrm{M}$ et al. Validation of an FFQ for evaluation of EPA and DHA intake. Public Health Nutr. 2009;12(10):1783-90.

41. Philibert A et al. Fish intake and serum fatty acid profiles from freshwater fish. Am J Clin Nutr. 2006;84(6):1299-307.

42. Oken $\mathrm{E}$ et al. Assessment of dietary fish consumption in pregnancy: comparing one-, four- and thirty-six-item questionnaires. Public Health Nutr. 2013: 1-11.

43. Farina M, Aschner M, Rocha JB. Oxidative stress in MeHginduced neurotoxicity. Toxicol Appl Pharmacol. 2011;256(3): 405-17.

44. Clarkson TW, Magos L. The toxicology of mercury and its chemical compounds. Crit Rev Toxicol. 2006;36(8):609-62.

45. Guzzi G, La Porta CAM. Molecular mechanisms triggered by mercury. Toxicology. 2008;244(1):1-12.

46. Kim SH, Johnson VJ, Sharma RP. Mercury inhibits nitric oxide production but activates proinflammatory cytokine expression in murine macrophage: differential modulation of NF-kappaB and p38 MAPK signaling pathways. Nitric Oxide. 2002;7(1):67-74.

47. Silva IA et al. Mercury exposure, malaria, and serum antinuclear/ antinucleolar antibodies in Amazon populations in Brazil: a crosssectional study. Environ Health. 2004;3(1):11.

48. Gardner RM, Nyland JF, Silbergeld EK. Differential immunotoxic effects of inorganic and organic mercury species in vitro. Toxicol Lett. 2010;198(2):182-90.

49. Gardner RM et al. Mercury exposure, serum antinuclear/ antinucleolar antibodies, and serum cytokine levels in mining populations in Amazonian Brazil: a cross-sectional study. Environ Res. 2010;110(4):345-54.

50. Nyland JF et al. Biomarkers of methylmercury exposure immunotoxicity among fish consumers in Amazonian Brazil. Environ Health Perspect. 2011;119(12):1733-8.

51. Silbergeld EK, IA Silva, JF Nyland. Mercury and autoimmunity: Implications for occupational and environmental health. Toxicol Appl Pharmacol. 2005; 207(2 SUPPL.): p. S282-S292.

52. Kim SH, Sharma RP. Mercury-induced apoptosis and necrosis in murine macrophages: Role of calcium-induced reactive oxygen species and p38 mitogen-activated protein kinase signaling. Toxicol Appl Pharmacol. 2004;196(1):47-57.

53. Kim SH, Sharma RP. Cytotoxicity of inorganic mercury in murine $\mathrm{T}$ and B lymphoma cell lines: Involvement of reactive oxygen species, $\mathrm{Ca} 2+$ homeostasis, and cytokine gene expression. Toxicol in Vitro. 2003;17(4):385-95.

54. Assefa $\mathrm{S}$ et al. Inorganic mercury exposure in prairie voles (Microtus ochrogaster) alters the expression of toll-like receptor 4 and activates inflammatory pathways in the liver in a sex-specific manner. Hum Exp Toxicol. 2012;31(4):376-86.

55. Okun E et al. Toll-like receptors 2 and 4 modulate autonomic control of heart rate and energy metabolism. Brain Behav Immun. 2014;36:90-100.

56. Woods JS et al. Genetic polymorphisms affecting susceptibility to mercury neurotoxicity in children: Summary findings from the Casa Pia Children's Amalgam Clinical Trial. Neurotoxicology. 2014.

57. Woods JS et al. Genetic polymorphisms of catechol-Omethyltransferase modify the neurobehavioral effects of mercury in children. J Toxicol Environ Health A. 2014;77(6):293-312.

58. Wang $\mathrm{Y}$ et al. An investigation of modifying effects of single nucleotide polymorphisms in metabolism-related genes on the relationship between peripheral nerve function and mercury levels in urine and hair. Sci Total Environ. 2012;417-418:32-8.

59. Julvez J et al. Prenatal methylmercury exposure and genetic predisposition to cognitive deficit at age 8 years. Epidemiology. 2013;24(5):643-50. 
60. Sztajzel J. Heart rate variability: a noninvasive electrocardiographic method to measure the autonomic nervous system. Swiss Med Wkly. 2004;134(35-36):514-22.

61. Scholte AJ et al. Cardiac autonomic neuropathy in patients with diabetes and no symptoms of coronary artery disease: comparison of 123I-metaiodobenzylguanidine myocardial scintigraphy and heart rate variability. Eur J Nucl Med Mol Imaging. 2010;37(9): $1698-705$.
62. Cygankiewicz I, Zareba W. Heart rate variability. Handb Clin Neurol. 2013;117:379-93.

63. Sheehan MC et al. Global methylmercury exposure from seafood consumption and risk of developmental neurotoxicity: a systematic review. Bull World Health Organ. 2014;92(4):254-269F. This global health review describes early-life mercury exposures and places in the context of possible neurotoxicity. 